\title{
Alcohol Levels Do Not Accurately Predict Physical or Mental Impairment in Ethanol-Tolerant Subjects: Relevance to Emergency Medicine and Dram Shop Laws
}

\author{
James R. Roberts • Denis Dollard
}

Published online: 1 April 2010

(C) American College of Medical Toxicology 2010

\begin{abstract}
The human body and the central nervous system can develop tremendous tolerance to ethanol. Mental and physical dysfunctions from ethanol, in an alcohol-tolerant individual, do not consistently correlate with ethanol levels traditionally used to define intoxication, or even lethality, in a nontolerant subject. Attempting to relate observed signs of alcohol intoxication or impairment, or to evaluate sobriety, by quantifying blood alcohol levels can be misleading, if not impossible. We report a case demonstrating the disconnect between alcohol levels and generally assigned parameters of intoxication and impairment. In this case, an alcoholtolerant man, with a serum ethanol level of $515 \mathrm{mg} / \mathrm{dl}$, appeared neurologically intact and cognitively normal. This individual was without objective signs of impairment or intoxication by repeated evaluations by experienced emergency physicians. In alcohol-tolerant individuals, blood alcohol levels cannot always be predicted by and
\end{abstract}

Dr. Roberts participates in alcohol-related litigation and Dr. Dollard does not participate in alcohol-related litigation.

There was no funding for this submission.

The information has not been previously presented.

J. R. Roberts $(\bowtie)$

Division of Toxicology, Department of Emergency Medicine,

Mercy Catholic Medical Center,

Philadelphia, PA, USA

e-mail: JRoberts@mercyhealth.org

D. Dollard

Emergency Department, Mercy Philadelphia Hospital,

501 South 54th Street,

Philadelphia, PA 19143, USA

e-mail: DDollard@mercyhealth.org do not necessarily correlate with outward appearance, overt signs of intoxication, or physical examination. This phenomenon must be acknowledged when analyzing medical decision making in the emergency department or when evaluating the ability of bartenders and party hosts to identify intoxication in dram shop cases.

\section{Introduction}

Physical and mental impairment from alcohol (ethanol) generally correlates with serum/blood alcohol concentration (BAC) and higher alcohol levels produce increased impairment and greater degrees of intoxication [1]. Published charts equate blood alcohol concentrations over 300 $400 \mathrm{mg} / \mathrm{dl}$ with inability to stand and walk, disorientation, confusion, stupor and coma, obvious overt intoxication, and circulatory and respiratory depression. Death from respiratory depression is referenced at alcohol concentrations above $450 \mathrm{mg} / \mathrm{dl}[2,3]$. The origins of these data are obscure, are poorly referenced, and do not account for tolerance or other circumstances.

The older non-emergency medicine literature has noted the phenomenon of acute and chronic alcohol tolerance; however, this well-recognized concept is not extensively reported or adequately emphasized in the recent literature [4-9]. The poor association of alcohol levels and outward sign of sobriety and the relevance of tolerance to emergency medicine have, however, been emphasized [1, 4]. The concept is generally absent in the lay press and poorly understood by the general public.

The presence of very high alcohol levels in patients who do not appear intoxicated is not uncommon in the emergency department (ED). Experienced clinicians know that measured alcohol levels and observed dysfunction can 
be greatly discordant in alcohol-tolerant individuals; and, impairment, or lack thereof, frequently does not correlate closely with alcohol measurements. The most recent edition of a major emergency medicine textbook has recently promulgated this axiom: "Chronic alcoholics can exhibit impressive tolerance. The blood alcohol concentration (BAC) of a person cannot be accurately determined without quantitative testing" [4].

Many alcohol-tolerant individuals function normally in daily society and the workplace, demonstrating little observable evidence of intoxication with alcohol levels touted as severely incapacitating or lethal. The capability to drive a motor vehicle with an alcohol level of 545 and $780 \mathrm{mg} / \mathrm{dl}$ has been documented [10, 11]. The concept of alcohol tolerance is of critical importance to clinicians who must initiate medical decisions based primarily on observations and clinical judgments of sobriety. Clinicians may be held accountable to published data strictly linking impairment to alcohol concentrations in the blood and are often forced to alter, institute, or change common sense interventions or reasonable medical plans because of these published standards. In fact, these are not standards at all, merely general guidelines that may be readily misrepresented by nonclinicians and in medicolegal milieus. The conundrum is also relevant for drinking establishments and party hosts who are liable for untoward events if they serve alcohol to someone visibly intoxicated.

To further elaborate more modern concepts of intoxication as it relates to alcohol blood levels and to cite a recent example of tremendous tolerance to alcohol, we report an ED patient with an extraordinary high serum alcohol level who displayed no evidence of physical or mental impairment during an extensive clinical examination by two clinicians. We believe this is the highest alcohol level reported in the recent emergency medicine/clinical toxicology literature exemplifying this phenomenon.

\section{Case Report}

A 35-year-old man presented to the ED by emergency medical services (EMS) after a witnessed generalized seizure. He was mildly confused at the scene, a condition attributed to a postictal state. Per EMS personnel, the man quickly regained a normal mental status during transport. In the ED, the patient relayed correct demographic information and a coherent past medical history. An underlying seizure disorder of unknown etiology was admitted, as was noncompliance to phenytoin. He had only one previous ED visit to this hospital for minor trauma after a prior seizure, but no alcohol level was obtained. Vital signs were: temperature $95.9^{\circ} \mathrm{F}$ orally, blood pressure $149 / 92 \mathrm{~mm} / \mathrm{Hg}$, pulse $100 / \mathrm{min}$, respirations $16 / \mathrm{min}$, and pulse oximetry
$100 \%$ on room air. Nursing triage described him as conscious and oriented in all spheres. The Glasgow Coma Scale was 15 . He admitted to "some" alcohol that morning. Upon physician evaluation $15 \mathrm{~min}$ after arrival, the patient had no medical complaints and asked for food. The initial physician evaluation revealed normal orientation, no slurred speech or nystagmus, normal cranial nerve function (II-XII), normal and equal strength and sensation, and normal finger-nose testing. The reflexes were normal and equal bilaterally, and there was no tremor or clonus. An odor of alcohol was noted on the breath. Within $20 \mathrm{~min}$, the blood pressure normalized. The seizure was attributed to an underlying seizure disorder of unknown cause and noncompliance to phenytoin. The plan was to observe the patient and obtain baseline blood tests. Following the initial evaluation, the man told the nurse he had to use the bathroom, and he independently got off the stretcher and walked $30 \mathrm{ft}$ to the bathroom unassisted. He demonstrated a completely normal gait to the first examining physician.

Blood drawn on admission demonstrated a serum alcohol level of $515 \mathrm{mg} / \mathrm{dl}$, reported by the laboratory as a "critical value." Since the laboratory report appeared so extraordinarily high when compared to the appearance and demeanor of the patient, we immediately repeated the alcohol level on a different blood sample taken at the same time. The repeat analysis yielded the same result. Reexamination by two experienced emergency physicians, with these data in mind, demonstrated no outward signs of alcohol intoxication, altered mental status, or neurological deficit. A repeat alcohol level $4 \mathrm{~h}$ later was $434 \mathrm{mg} / \mathrm{dl}$, demonstrating appropriate kinetics. Except for a mildly depressed potassium $(3.1 \mathrm{mmol} / \mathrm{l})$ and a mild anemia (hemoglobin $11.6 \mathrm{~g} / \mathrm{dl}$ ), basic laboratory tests were normal, including liver enzymes, amylase, and lipase. The phenytoin blood level was nondetectable, and a loading dose of phenytoin was administered. Four hours after arrival, the patient ate a full meal and remained independently ambulatory, normally conversant, and neurologically normal to three subsequent physician evaluations. Despite wishes to leave the ED after eating a meal, he was not allowed to do so because of the laboratory perturbation. Vital signs remained normal, no additional medical conditions were discovered, and he did not experience another seizure. The patient was treated with prophylactic oxazepam (30 mg every $8 \mathrm{~h}$ ), but alcohol withdrawal was not evident during the hospitalization. Mild abdominal pain developed, but no cause was found, and he became asymptomatic in 2 days. He declined further evaluation or treatment and was discharged 3 days later to "go to work," occupation not forthcoming. The patient was lost to followup. It was concluded that this man experienced a breakthrough seizure, underlying etiology undetermined, 
exacerbated by medication noncompliance and alcohol abuse.

\section{Discussion}

Although perhaps not cognizant of the degree of alcohol tolerance possible or the nuances of blood alcohol testing, the lay public has a general intuitive understanding of the issue of tolerance, described as "being able to hold one's liquor." Usually ascribed to only hard-core alcoholics, there is evidence that significant tolerance can develop within 45 days of consecutive drinking, even in social drinkers [12]. Numerous reports have proven that human response to alcohol is quite variable and unpredictable $[13,14]$. The effect of alcohol on psychomotor performance and perceived impairment in chronic heavy drinkers is a complex subject, poorly studied, and not yet fully understood. Repeated exposure to alcohol may result in not only physiologic tolerance but behavioral tolerance, a neuroadaptive process resulting in a diminished behavioral response to a given alcohol level. While controlled studies of light to moderate drinkers have demonstrated the alcohol tolerance phenomenon, there has been scant scientific investigation of subjects who are chronic heavy drinkers who appear to have massive alcohol tolerance [15-18]

The behavioral and subjective effects from any given alcohol level vary widely in the general population. Prior studies have demonstrated that moderate female drinkers of alcohol (about 35 drinks per month) demonstrated a reduced response to a given amount of alcohol ingestion compared to light drinkers (about seven drinks per month) and were viewed as less impaired following similar alcohol consumption. This was evident even among subjects who did not meet criteria for alcohol abuse [15]. While there is a general dose-dependent effect of alcohol in humans with regard to diminished cognitive and psychomotor skills, prior experience with alcohol, duration and degree of consumption, and individual tolerance render impossible a strict assignment of impairment or degradation of physical, mental, and cognitive parameters to any given alcohol level.

Case reports and small series have reported apparently normal or medically stable patients who have significantly elevated alcohol levels. The older literature is replete with examples of this clinical observation [19-25]. As examples, chronic alcohol abusers have survived alcohol poisoning with levels as high as $1,510 \mathrm{mg} / \mathrm{dl}$ [19]. Several authors in the 1980s reported markedly elevated alcohol concentrations in alcoholics who appeared sober, but specific details were lacking. Sullivan et al. and Watanabe et al. vaguely described individuals with alcohol levels of 1,510 and $1,121 \mathrm{mg} / \mathrm{dl}$, respectively, who were "confused but alert" $[20,21]$. Redmond reported a patient capable of "articulate conversation" with a BAC of $897 \mathrm{mg} / \mathrm{dl}$ [5]. Urso et al. described a subject who appeared "quite sober" despite an alcohol level of $540 \mathrm{mg} / \mathrm{dl}$ [22]. Perper et al. described alcoholics at a voluntary detoxification center, with alcohol levels as high as $444 \mathrm{mg} \%$, demonstrating seeming minor outward effects [23]. Lindbald and Olsson described 24 patients whose blood level exceeded $500 \mathrm{mg} / \mathrm{dl}$, while being either awake or roused by nonpainful stimuli [24]. Davis and Lipson noted very little evidence of intoxication in 18 patients with blood levels in the range of 300 $450 \mathrm{mg} / \mathrm{dl}$ [25]. Finally, Redmond recorded a blood alcohol level of $897 \mathrm{mg} / \mathrm{dl}$ in a patient capable of articulate conversation, $4 \mathrm{~h}$ after cessation of drinking [6]. This author also commented upon 20 individuals who "were responsive and able to give a good account of themselves" with blood alcohol levels above $500 \mathrm{mg} / \mathrm{dl}$.

Numerous states have so-called dram shop laws, a moniker coined from the eighteenth century English business custom where gin was sold by the spoonful, called a dram. Under this concept, a legal liability may be assigned to those who provide alcoholic beverages to already intoxicated individuals who then subsequently injure themselves or cause harm to others due to their intoxicated state. Untoward events addressed by such laws include personal injury, loss of financial support, and property damage. Dram shop statutes usually refer to restaurants, bars, and other retail establishments that are licensed to serve alcoholic beverages. However, the concept has also been applied to party hosts and other alcohol servers who have provided alcohol to an intoxicated person, when injury to that person or third parties has resulted from the actions of the intoxicated subject. Laws vary from state to state, and there are varying degrees of liability for alcohol servers (http://www.cga.ct.gov/2005/rpt/2005-R-0922.htm).

Generally, dram shop financial liability is considered when alcohol was furnished to an individual already demonstrating obvious intoxication or under circumstance whereby alcohol impairment is manifested or should have been known. Ostensibly, it should have been clear that a customer or partygoer was visibility intoxicated and therefore a potential danger to himself/herself or others from additional alcohol. While the responsible entity is expected to refuse to provide additional alcohol to an obviously intoxicated subject, such a mandate requires that a layperson be able to assess impairment by outward sign and actions. Since sobriety and alcohol levels may not correlate, this law has been questioned and termed archaic, and it has been posited that the judgment legally required of bartenders is unreasonable [26].

The difficulty for even experienced physicians to use outward signs of intoxication to identify or intuit a quantitative alcohol level traditionally defining severe intoxication is intuitively obvious, but studies have con- 
cluded that police officers, social drinkers, trained interviewers, mental health counselors, and alcohol counselors are often unable to recognize when others are intoxicated [27-29]. When police officers give estimates of alcohol levels, the error is usually an underestimation of blood levels or failure to detect alcohol effect [28]. In headinjured patients, physicians cannot clinically discern a change in the Glasgow Coma Scale until the blood alcohol level is over $200 \mathrm{mg} / \mathrm{dl}$ [30]. The task for a party host or bar tender is even more challenging.

As demonstrated by this case, the lack of strict concordance of quantitative alcohol blood testing and outward signs of intoxication calls into question the value of alcohol testing with regard to clinical or social decision making. It is also obvious that assigning a level of impairment or degree of intoxication based on blood alcohol quantification alone is a flawed concept in the alcohol-tolerant individual.

\section{Conclusion}

We concluded that our patient demonstrated relatively common, but greatly underappreciated, massive tolerance to alcohol, by demonstrating a totally normal mental status and neurological examination with an alcohol level commonly reported in medical literature to be lethal [31, 32]. While there is a general dose-dependent effect of alcohol on psychomotor and cognitive impairment, alcohol levels alone do not define impairment and cannot be used to assign a strict level of intoxication in a tolerant individual. In tolerant individuals, alcohol levels provide minimal quantitative information and cannot be used, in isolation, to define clinical impairment or intoxication. This phenomenon must be taken into consideration when analyzing situations where a patient's alcohol blood level alone is retrospectively used to assess a clinician's clinical decision making. There are very few data demonstrating actual clinical utility of alcohol testing. The routine practice of drawing alcohol levels in the ED and using the results to draw a strict clinical inference on the degree of impairment or to dictate medical interventions of an individual who is tolerant to alcohol seems a useless endeavor.

In addition, to conclude that a subsequent alcohol level is per se evidence of serving alcohol to an obviously intoxicated patron or party guest is faulty analysis. Surely, bartenders or party hosts would have a difficult, if not impossible, task of estimating laboratory-defined impairment by outward appearance if subsequently such impairment is based solely on alcohol levels.

If experienced emergency physicians cannot reliably predict alcohol levels based on outward appearance, how could a bartender or party host be expected to do so?

\section{References}

1. Yip L (2006) Ethanol. In: Flomenbaum N, Goldfrank L, Hoffman R, Howland MA, Lewin N, Nelson L (eds) Goldfrank's toxicologic emergencies, 8th edn. McGraw-Hill, New York, pp 1153-1154

2. Garriott JC (2008) In: Garriott JC (ed) Garriott's medicolegal aspects of alcohol, 5th edn. Lawyers \& Judges, Tucson, p 28

3. USDHHS, National Institute on Alcohol Abuse and Alcoholism (1998) Fourth special report to Congress on alcohol and health. In: Goldfrank LR, Flomenbaum NE, Lewin NA, Weisman RS, Howland MA, Hoffman RS (eds) Goldfrank's toxicologic emergencies, 6th edn. Appleton \& Lange, Norwalk, p 1027

4. Finnell JT, McMicken DB (2009) In: Marx J, Hockberger R, Walls R, Adams JG et al (eds) Rosen's emergency medicine: concepts and clinical practice, 7th edn. Mosby/Elsevier, Philadelphia, p 2372

5. Redmond AD (1986) Central nervous system depression and high blood ethanol levels. Lancet 327(8484):805

6. Redmond AD (1983) Blood alcohol concentrations and conscious level. Alcohol Alcoholism 18:89-91

7. Goldberg L (1943) Quantitative studies on alcohol tolerance in man. Acta Physiol Scand 5(suppl 16):1

8. deWit H, Uhlenhuth EH, Pierri J et al (1987) Individual differences in behavior and subjective responses to alcohol. Alcohol Clin Exp Res 11:51-59

9. Brick J, Erickson CK (2009) Intoxication is not always visible: an unrecognized prevention challenge. Alcohol Clin Exper Res 33:1489-1507

10. Jones AW (1999) The drunkest drinking driver in Sweden: blood alcohol concentration $0.545 \% \mathrm{w} / \mathrm{v}$. J Stud Alc 60:400-406

11. Hammond KB, Rumack BH, Rodgerson DO (1973) Blood ethanol: a report of unusually high levels in a living patient. JAMA 226:63-64

12. Bennett RH, Cherek DR, Spiga R (1993) Acute and chronic alcohol tolerance in humans: effect of dose and consecutive days of exposure. Alcohol Clin Exp Res 17:740-745

13. Hiltunen AJ (1997) Acute alcohol tolerance in cognitive and psychomotor performance: influence of the alcohol dose and prior alcohol experience. Alcohol 14:125-130

14. Marczinski CA, Fillmore MT (2009) Acute alcohol tolerance on subjective intoxication and simulated driving performance in binge drinkers. Psychol Addict Behav 2:238-247

15. Evans S, Levin FR (2004) Differential response to alcohol in light and moderate drinkers. Behav Pharmcol 15:167-181

16. Brumback T, Cao D, King A (2007) Effects of alcohol on psychomotor performance and perceived impairment in heavy binge social drinkers. Drug Alcohol Depend 9:10-17

17. Fillmore MT, Vogel-Sprott M (1996) Social drinking history, behavioral tolerance and the expectation of alcohol. Psychopharmacology (Berl) 127:359-365

18. Fillmore MT, Sprott-Vogel M (1995) Behavioral effects of alcohol in novice and experienced drinkers: alcohol expectancies and impairment. Psychopharmacology (Berl) 122:175-184

19. Johnson RA, Noll EC, Rodney WM (1982) Survival after a serum ethanol concentration of $1 \frac{1 / 2}{2} \%$. Lancet 2:1394

20. Sullivan JB Jr, Hauptman M, Bronstein AC (1987) Lack of observable intoxication in humans with high plasma alcohol concentration. J Forensic Sci 32:1660-1665

21. Wantanabe A, Kobayash M, Hobara BS et al (1985) A report of unusually high blood ethanol and acetaldehyde levels in two surviving patients. Alcoholism Clin Exp Res 9:14-16

22. Urso T, Gavaler JS, Van Thiel DH (1981) Blood ethanol levels in sober alcohol users seen in an emergency room. Life Sci 28:10531056 
23. Perper JA, Twerski A, Wienand JW (1986) Tolerance at high blood alcohol concentrations: a study of 110 cases and review of the literature. J Forensic Sci 31:212-221

24. Lindbald B, Olsson R (1976) Unusually high levels of blood alcohol. JAMA 236:1600-1602

25. Davis AR, Lipson AH (1986) Central nervous system tolerance to high blood alcohol levels. Med J Aust 144:9-12

26. Langenbucher JW, Nathan PE (1983) Psychology, public policy, and the evidence for alcohol intoxication. Am Psychol 38:10701077

27. Brick J, Carpenter JA (2001) The identification of alcohol intoxication by police. Alcohol Clin Exp Res 25:850-855
28. Moskowitz H, Burns M, Ferguson S (1999) Police officers' detection of breath odors from alcohol ingestion. Accid Anal Preven 31:175-180

29. Carroll N, Rosenberg H, Funke S (1988) Recognition of intoxication by alcohol counselors. J Subs Abuse Treat 5:239-246

30. Galbraith S, Murray WR, Patel AR (1976) The relationship between alcohol and head injury and its effect on the conscious level. Br J Surg 63:128-130

31. Dubowski KM (1980) Alcohol determination in the clinical laboratory. Am J Clin Pathol 74:747

32. Yost DA (2002) Acute care for alcohol intoxication. Postgrad Med 112:14-26 